\title{
Numerical Information Retrieval and Modeling for Small Droplet Samples
}

\author{
P.He ${ }^{1}$, L.Xue ${ }^{1}$, Y.Qi $^{1}$, L.Zhang $^{1}$, Y.Lu ${ }^{2}$ \\ ${ }^{1}$ Bioengineering Department, Zhengzhou Univ. 100 Science Avenue, Zhengzhou 450052, P.R.China \\ ${ }^{2}$ Henan Institute of Occupational Diseases, Zhengzhou 450052, P.R.China \\ gp268@hotmail.com
}

\begin{abstract}
Information retrieval is applied widely in modern digital world. Particularly in chemistry and life science, retrieval technology is used for raw data acquirement, analytical modeling and processing optimization. The complicated microdroplets fabrication can be facilitated through different retrievals. In this work, different information retrieval and analysis were performed through small samples. The linear model could depict general droplet size variation. Nonlinear item was found and then added in this model to ameliorate its effectiveness. The biomicrofluidic droplet variations, as complicated procedure, were described satisfactorily in the optimized nonlinear model. Neural Networks Modeling (NNM) was performed to train the small samples and finally the verification of NNM demonstrated its accuracy for small droplet samples.

Index Terms - Information retrieval, Data analysis,
\end{abstract} Biomicrofluidics, Droplets, Modeling.

\section{Introduction}

The information processing through numerical information retrieval technology facilitated and broadened the research and application of known raw data. The complicated micro-droplets fabrication turned to be easy through different retrieval technologies. The combination of microfluidics with life and medical science permitted to efficiently realize biotechnological monodisperse droplets, applied widely in industries as well as sciences [1]. The micrometer-scale droplets with size homogeneity provided same compartments to realize drug targeting and discovery [2] and food emulsions [3]. These compartments make it accessible to compare the growth and differentiation between confined cells. Biomicrofluidics involving different fundamental disciplines is a complex but promising topic for future investigation and application. Experimental advances of fluid components or systems at micrometer scale based on microfabrication technologies permitted the involvement of different microfluidic valves, pumps and flow sensors into main microfluidic geometry [4]. In spite of the wide-spread use of biomicrofluidic droplets, information retrieval and numerical modeling on droplet size variation were still in progress. Numerical modeling based on simple flow theory was reported to describe microfluidics. The flow profile and the liquid crystal orientational profile in microfluidic devices characterized by cross-section showed three distinct regimes of weak, medium, and strong flow with variation of driving pressure. Numerical modeling solutions were compared with solutions from optical microscopy [5]. Specifically, a modeling presented the viscous forces as well as capillary effects on the droplet generation. For the modeling on different droplet size evolution under biomicrofluidic environment, two typical geometries should be introduced: T-shaped series and flow-focusing series. Descriptive modeling for droplet fluidic phenomena under T-shaped microfluidic confinement explained the transition regimes of droplet formation, by incorporation of geometrical parameters and the force balance [6]. However, flow-focusing microfluidics is the other typical geometry. Through numerical retrieval technology on small droplet samples at this geometry, reasonable models can be extracted to readily describe its variation.

\section{II . Information Analysis and Retrieval}

\section{A. Experiment design}

The experiments were designed to test the possible combinations of flow rates, in order to clarify the role of each parameter. The central flow rate $\mathrm{Q}_{\mathrm{c}}$ was designed to include three levels, with a specific value each, as shown in Table 1; the lateral flow rates $Q_{1}$ consisted of three levels, each level with a particular value, shown in Table 2. Experiment conditions were distributed uniformly in the testing space, shown in Fig. 1. This balanced design led to easy result comparison.

TABLE 1 Three $Q_{c}$ levels for corresponding values

\begin{tabular}{|c|c|c|c|}
\hline level & 1 & 2 & 3 \\
\hline value & 3.4 & 6.6 & 9.6 \\
\hline
\end{tabular}

TABLE 2 Three $Q_{1}$ levels for corresponding values

\begin{tabular}{|c|c|c|c|}
\hline level & 1 & 2 & 3 \\
\hline value & 10 & 15 & 20 \\
\hline
\end{tabular}

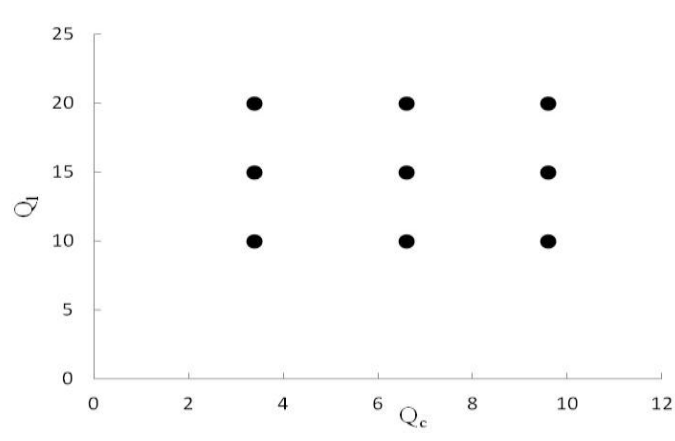

Fig. 1. Scattering of $\mathrm{Q}_{c}$ and $\mathrm{Q}_{1}$ in research space 


\section{B. Value analysis}

Value analysis was undertaken to see the effects of $Q_{c}$ and $\mathrm{Q}_{1}$. The results presented the relation between droplet size $\mathrm{S}$ as function of $\mathrm{Q}_{\mathrm{c}}$ and $\mathrm{Q}_{1}$ in biomicrofluidics. $\mathrm{S}$ seemed to be on direct proportion to $\mathrm{Q}_{\mathrm{c}}$, while almost on reverse proportion to $\mathrm{Q}_{1}$. This experimental observations can be logically explained: for increase of $\mathrm{Q}_{c}$, the central viscous force became stronger, which took effects to give more time for droplet formation, permitting to enlarge droplet size; however, the augmentation of $\mathrm{Q}_{1}$ led to enforce lateral shear stress on central flow, allowing for reduction of droplet sizes. We find the variation of droplet size for $\mathrm{Q}_{\mathrm{c}}$ is one-fifth as large as that of $\mathrm{Q}_{1}$. The $\mathrm{Q}_{1}$ seemed to weigh more for droplet size variation in the process.

\section{Correlation analysis}

The correlation analysis was performed between $\mathrm{Q}_{\mathrm{c}}$ and $\mathrm{Q}_{1}$.. $\mathrm{Q}_{\mathrm{c}}$ and $\mathrm{Q}_{1}$ were found to be independent to each other, with $\mathrm{R}^{2} \approx 0$. This is a logic result for the flow rates adjustment are separately controlled between $Q_{c}$ and $Q_{1}$. This finding proposed a possibility to include these two flow rates into the mathematical model to express their linear behaviors for droplet generation.

\section{Modeling}

As shown in Equation 1, the linear model showed that as increase of $\mathrm{Q}_{\mathrm{c}}$ or decrease of $\mathrm{Q}_{1}$. droplet sizes increase. The same phenomena were observed through different experiments. This result could be explained through mechanical viewpoint: In this biomicrofluidic environment, surface tension and viscous force take main effects. Surface tension between two flows tends to intercept flow elongation and facilitate the droplet formation, while the fluidic viscous force takes more effects on keeping flow stable. The capillary number $\mathrm{Ca}$ presented the ratio of viscous effects to surface tension. In this study, the $\mathrm{Ca} \sim O\left(10^{-1}\right)$, the surface tension overweighed the viscous force. This led to the formation of different droplets. Besides, the greater central flow rate $Q_{c}$ supported stronger viscous effects, permitting to extend the central flow much longer; while with augmentation of lateral flow rate $Q_{1}$., the shear stress on the central flow become stronger, resulting in smaller droplets. The same phenomena were observed through different experiments.

$$
\mathrm{S}=28 \mathrm{Q}_{\mathrm{c}}-33 \mathrm{Q}_{1}+824, R^{2}>0.8 \text {. }
$$

However, this linear model seemed to some extent to express droplet generation, but $R^{2}$ value is something short of effectiveness. The droplet generation is regarded as nonlinear process involving the interactions between pressures or flowrates control, design of biomicrouidic environment, and chemical interfacial compatibility. Then the nonlinear technique was used to optimize the modeling. The flow product item $\left(\mathrm{Q}_{\mathrm{c}} \times \mathrm{Q}_{\mathrm{l}}\right)^{0.5}$ was tested in the linear model to see the interaction between the two flow ratios. We added the nonlinear item $\left(\mathrm{Q}_{\mathrm{c}} \times \mathrm{Q}_{\mathrm{l}}\right)^{0.5}$ to the model to involve the interaction effects.

\section{E. Nonlinear Optimization}

The modeling for droplet generation is optimized with inclusion of flow rates item $\left(\mathrm{Q}_{\mathrm{c}} \times \mathrm{Q}_{\mathrm{l}}\right)^{0.5}$, as presented in Equation. 2. The improved nonlinear model could explain well the droplet size variation.

$$
S=168 Q_{c}+26 \mathrm{Q}_{1}-181\left(\mathrm{Q}_{\mathrm{c}} \times \mathrm{Q}_{\mathrm{l}}\right)^{0.5}+753, R^{2}>0.91
$$

This nonlinear model showed that as the increase of $\mathrm{Q}_{\mathrm{c}}$ or $\mathrm{Q}_{1}$, the droplet sizes increase; besides, with the increase of flow rates item $\left(\mathrm{Q}_{\mathrm{c}} \times \mathrm{Q}_{\mathrm{l}}\right)^{0.5}$, droplet sizes varied towards the opposite direction. For $\mathrm{Q}_{c}$, droplet sizes varying as the same results as linear model; For $\mathrm{Q}_{1}$, however, droplet sizes varied differently comparing with linear model. This difference consisted in the inclusion of nonlinear item $\left(\mathrm{Q}_{\mathrm{c}} \times \mathrm{Q}_{\mathrm{l}}\right)^{0.5}$, showing that the interaction effects could take effects on each items at the model. The addition of nonlinear item improved the model quality but also expressed the descriptive complexity for the droplet variation. The conclusion deduced from this model coincided well with the experimental observations.

\section{Neural Network Modeling and Verification}

The Neural Network Modeling (NNM) for droplet size variation was performed. This nonlinear model contains input layer, invisible neural nodes and responses. $\mathrm{Q}_{\mathrm{c}}$ and $\mathrm{Q}_{\mathrm{l}}$ were contained in the input layer, while the response represented the droplet size. The theoretical analysis was performed to compare the values extracted from experiments with the theoretical ones calculated through neural networks modeling. The validated results were presented in the Fig. 2. These values fell in the vicinity of butted baseline, demonstrating the effectiveness of this modeling based on artificial neural network technology.

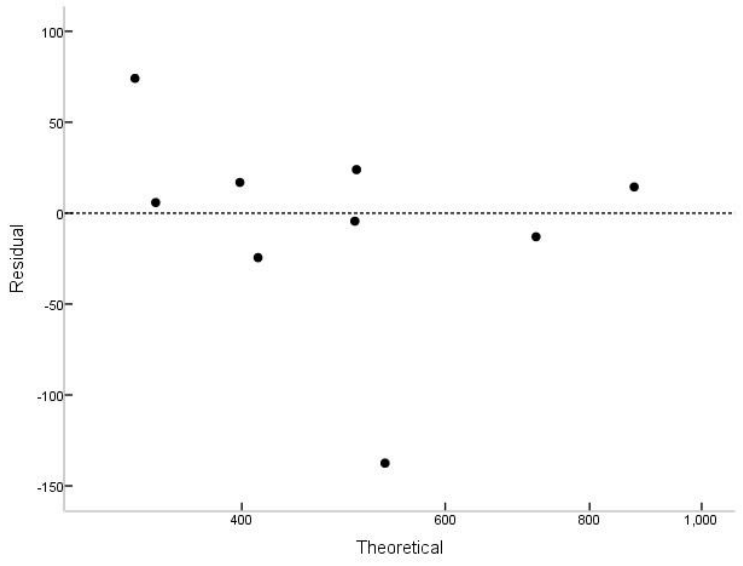

Fig. 2.Verification of Neural Network Modeling (NNM) 


\section{IV . Conclusion}

Several information retrieval technologies were applied to small droplet samples. The role for two independent factors $Q_{c}$ and $\mathrm{Q}_{1}$ were analysed. The latter factor weighed more than the former for droplet enlargement in this process. Then linear model on droplet size variations was established to present droplet generation process; but this linear model is to some extent lack of descriptive satisfaction. The nonlinear item was introduced to achieve improved descriptive quality. The NNM technique was applied for droplet information retrieval. The verification procedure demonstrated optimized nonlinear model as well as NNM can well retrieve information through small droplets samples.

\section{V . Acknowledgment}

This work was supported by grant at Zhengzhou University.

\section{References}

[1] Shia-Yen Teha, Robert Lina, Lung-Hsin Hungb and Abraham P. Lee, "Droplet microfluidics," Lab Chip, vol 8, pp 198-220, 2008.

[2] Petra S. Dittrich and Andreas Manz, "Lab-on-a-chip: microfluidics in drug discovery," Nature Reviews Drug Discovery, vol 5, pp 210-218, March 2006.

[3] George A. van Aken, "Relating Food Emulsion Structure and Composition to the Way It Is Processed in the Gastrointestinal Tract and Physiological Responses:What Are the Opportunities?" Food Biophysics, vol 5, pp. 258-283, 2010.

[4] P Gravesen, J Branebjerg and O S Jensen, "Microfluidics-a review," B. J. Micromech. Microeng., vol 3 pp.168, 1993.

[5] Anupam Sengupta1, Uros Tkalec, Miha Ravnik, Julia M. Yeomans, Christian Bahr, and Stephan Herminghaus, "Liquid Crystal Microfluidics for Tunable Flow Shaping," Phys. Rev. Lett, vol 110, pp. 048303048307, 2013.

[6] Tomasz Glawdel, Caglar Elbuken, and Carolyn L. Ren, "Droplet formation in microfluidic T-junction generators operating in the transitional regime. II. Modeling," Phys. Rev. E, vol 85, pp. 016323016334, 2012. 